\title{
Improved gluonic actions on anisotropic lattices
}

\author{
Colin Morningstar ${ }^{\mathrm{a}}$

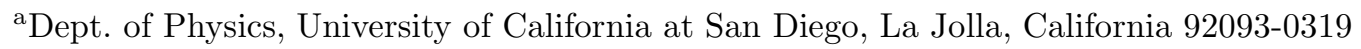

The use of novel perturbatively-improved gluonic actions on anisotropic lattices in which the temporal spacing is much smaller than that in the spatial directions is discussed. Such actions permit more efficient measurements of noisy correlation functions, such as glueball correlators, on coarse lattices. A derivation of these actions at tree-level is outlined; mean-field link renormalization plays a crucial role in their construction. Results for the low-lying glueball masses and the heavy-quark potential are presented.

The use of improved actions makes possible accurate Monte Carlo simulations of QCD on coarse lattices with greatly reduced computational effort. However, for some calculations, such as glueball masses [1], the coarseness of the temporal lattice spacing can be a severe drawback, greatly reducing the number of correlator time intervals which can be measured. This problem can be circumvented by using anisotropic lattices in which the temporal spacing is much smaller than that in the spatial directions, enabling one to exploit the enhanced signal-to-noise of the correlation functions at smaller temporal separations. Improved lattice actions often admit spurious states with energies of order $1 / a$, where $a$ is the lattice spacing. On fine grids, such modes are comfortably high-lying and little affect the low-lying modes of interest. However, on a coarse lattice, this may no longer be true. Reducing the temporal spacing also cures this problem by elevating the spurious states to higher energies of order $1 / a_{t}$, where $a_{t}$ is the temporal lattice spacing. The lifting of spurious states is particularly important when designing improved fermion actions [2]. In fact, anisotropic lattices will be useful whenever one is faced with a four-momentum in which one component is unusually large, such as in the calculation of glueball masses 3], the heavy quarkonium spectrum, or hadronic form factors at large momentum transfers. Anisotropic lattices have also long been used in finite temperature studies.

To remove $O\left(a^{2}\right)$ errors from all Green's functions in lattice QCD at tree level in perturbation theory, one needs only to eliminate $O\left(a^{2}\right)$ arti- facts in the lattice action. This can be done by performing a small- $a$ expansion of some suitable lattice action and adjusting the interaction couplings so that the leading terms in the expansion reproduce the correct continuum QCD action and the $O\left(a^{2}\right)$ terms are absent. Any lattice operator $W_{\beta}[U]$ constructed from link variables will have a small- $a$ expansion of the form

$W_{\beta}[U]=\sum_{k=0}^{\infty} \int d^{4} x \xi^{-1} a_{s}^{k-4} \sum_{\alpha=1}^{M(k)} c_{\alpha}^{(k)} Q_{\alpha}^{(k)}(x)$,

where $c_{\alpha}^{(k)}$ are the expansion coefficients, the spatial lattice spacing is $a_{s}$, the temporal spacing is $a_{t}=\xi a_{s}$, and $Q_{\alpha}^{(k)}(x)$ are local, dimension- $k$ continuum operators at point $x$ which are invariant under gauge transformations and all symmetries of the anisotropic lattice. There are no such operators for dimensions less than four. There are only two dimension-four operators: $Q_{1}^{(4)}=$ $g^{2} \operatorname{Tr} \mathbf{E}^{2}$ and $Q_{2}^{(4)}=g^{2} \operatorname{Tr} \mathbf{B}^{2}$, where $\mathbf{E}$ and $\mathbf{B}$ are the chromoelectric and chromomagnetic fields, respectively. There are no dimension-five operators, but there are 18 independent dimension-six operators, ten of which may be expressed as unimportant total derivatives. Thus, we adjust the couplings of the $W_{\beta}[U]$ lattice operators so that the coefficients of the eight dimension-six operators vanish and the coefficients of the two dimensionfour operators equal each other. If we are interested only in on-shell improvement, we are also free to use a field redefinition to set some of the coefficients of the dimension-six operators to zero.

Perturbation theory by itself does not reliably 
determine the couplings in the improved action. It is known that a judicious combination of perturbation theory and mean field theory works much better. Mean field theory is introduced by renormalizing the link variables: $U_{j}(x) \rightarrow$ $U_{j}(x) / u_{s}$ and $U_{t}(x) \rightarrow U_{t}(x) / u_{t}$, where $u_{t}$ and $u_{s}$ are the mean values of the gluon link operators for the temporal and spatial links, respectively.

Carrying out the above procedure, one of the simplest actions we can arrive at is given by [4]:

$$
\begin{aligned}
S_{I}[U] & =\beta \xi\left\{\frac{5}{3 u_{s}^{4}} W_{\mathrm{sp}}+\frac{5}{3 \xi^{2} u_{s}^{2} u_{t}^{2}} W_{\mathrm{tp}}\right. \\
& -\frac{1}{12 u_{s}^{6}} W_{\mathrm{sr}}-\frac{1}{12 \xi^{2} u_{s}^{4} u_{t}^{2}} W_{\mathrm{str}} \\
& \left.-\frac{1}{12 \xi^{2} u_{s}^{2} u_{t}^{4}} W_{\mathrm{ttr}}\right\},
\end{aligned}
$$

where $\beta=6 / g^{2}, W_{c}=\sum_{c} \frac{1}{3} \operatorname{Re} \operatorname{Tr}\left(1-U_{c}\right)$, and $U_{\mathrm{sp}}$ denotes the spatial plaquettes, $U_{\mathrm{tp}}$ indicates the temporal plaquettes, $U_{\mathrm{sr}}$ denotes the product of link variables about a planar $2 \times 1$ spatial rectangular loop, $U_{\text {str }}$ refers to the short temporal rectangles (one temporal spacing, two spatial), and $U_{\mathrm{ttr}}$ refers to the tall temporal rectangles (two temporal spacings, one spatial). The mean values $u_{t}$ and $u_{s}$ are determined by guessing input values for use in the action, measuring the mean links in a simulation, then readjusting the input values accordingly. When $a_{t}$ is significantly smaller than $a_{s}$, we expect the mean temporal link $u_{t}$ to be very close to unity. For example, in Landau-gauge perturbation theory, $1-\left\langle\frac{1}{3} \operatorname{Tr} U_{t}\right\rangle \propto$ $\left(a_{t} / a_{s}\right)^{2}$. Hence, to simplify matters, we set $u_{t}=1$. A convenient and gauge-invariant definition for $u_{s}$ in terms of the mean spatial plaquette is then given by $u_{s}=\left\langle\frac{1}{3} \operatorname{Re} \operatorname{Tr} P_{s s^{\prime}}\right\rangle^{1 / 4}$, where $P_{s s^{\prime}}$ denotes the spatial plaquette.

One defect of the action $S_{I}$ is that the gluon spectrum has spurious high-energy states arising from $W_{\mathrm{ttr}}$, which spans two time slices. These modes occur at energies of order $2 / a_{t}$ and have little effect on simulation results, but they can cause problems when applying the variational method to extract masses from short-time correlation functions. These modes may be eliminated by relaxing the improvement conditions, requiring that the coefficients of all dimension-six oper-
Table 1

Renormalization of the anisotropy. The input anisotropy is $\xi=a_{t} / a_{s}$, and $\xi_{\text {meas }}$ is the measured anisotropy (see text). The lattice spacing $a_{s}$ is set using the $1 P-1 S$ splitting in charmonium for $S_{3}$ and the string tension for $S U_{2} . S_{L W}$ is the improved Lüscher-Weisz action [5].

\begin{tabular}{cccll}
\hline Action & $\beta$ & $\xi$ & $\xi_{\text {meas }}$ & $a_{s}(\mathrm{fm})$ \\
\hline$S_{L W}$ & 1.9 & $1 / 1$ & 1.00 & $0.397(4)$ \\
$S_{I I}$ & 2.0 & $1 / 2$ & $0.505(11)$ & $0.418(6)$ \\
& 2.0 & $1 / 3$ & $0.342(10)$ & $0.372(6)$ \\
& 2.3 & $1 / 2$ & $0.507(10)$ & $0.311(5)$ \\
$S_{I}$ & 2.0 & $1 / 2$ & $0.505(10)$ & $0.371(4)$ \\
& 2.3 & $1 / 2$ & $0.510(11)$ & $0.271(3)$ \\
$S_{I I}\left(u_{s}=1\right)$ & 3.9 & $1 / 2$ & $0.40(2)$ & $0.40(2)$ \\
$S_{I I}\left(S U_{2}\right)$ & 0.848 & 0.276 & $0.286(6)$ & $0.371(9)$ \\
& 1.027 & 0.351 & $0.357(4)$ & $0.283(6)$ \\
& 1.114 & 0.409 & $0.417(6)$ & $0.249(6)$ \\
\hline
\end{tabular}

ators except $\operatorname{Tr}\left[\left(D_{t} \mathbf{E}\right)^{2}\right]$ vanish. One then obtains:

$$
\begin{aligned}
S_{I I}[U] & =\beta \xi\left\{\frac{5}{3 u_{s}^{4}} W_{\mathrm{sp}}+\frac{4}{3 \xi^{2} u_{s}^{2} u_{t}^{2}} W_{\mathrm{tp}}\right. \\
& \left.-\frac{1}{12 u_{s}^{6}} W_{\mathrm{sr}}-\frac{1}{12 \xi^{2} u_{s}^{4} u_{t}^{2}} W_{\mathrm{str}}\right\}
\end{aligned}
$$

This action has $O\left(a_{t}^{2}\right)$ errors which are very small, being suppressed by a factor of $\xi^{4}$. For this action, various values for the parameter $u_{s}(\beta, \xi)$ are $u_{s}\left(1.7, \frac{1}{3}\right)=0.745, u_{s}\left(2.0, \frac{1}{3}\right)=0.772$, and $u_{s}\left(2.4, \frac{1}{3}\right)=0.806$.

The renormalization of the anisotropy can be determined by measuring the static-quark potential $V(x, y, z)$ from Wilson loops in different orientations. For example, Wilson loops in the $x t$ and $x y$ hyperplanes have the following asymptotic behaviours ( $I, J$ positive integers):

$$
\begin{aligned}
W_{x t}\left(I a_{s}, J a_{t}\right) & \stackrel{J \rightarrow \infty}{\longrightarrow} Z_{x t} e^{-J a_{t} V\left(I a_{s}, 0,0\right)} \\
W_{x y}\left(I a_{s}, J a_{s}\right) & \stackrel{J \rightarrow \infty}{\longrightarrow} Z_{x y} e^{-J a_{s}\left[V\left(I a_{s}, 0,0\right)+V_{0}\right]}
\end{aligned}
$$

where $Z_{x t}, Z_{x y}$, and $V_{0}$ are renormalization constants. From the asymptotic behaviour of $W_{x t}$ we obtain $\Delta_{x t}=a_{t}\left[V\left(2 a_{s}, 0,0\right)-V\left(a_{s}, 0,0\right)\right]$ and from $W_{x y}$ we obtain $\Delta_{x y}=a_{s}\left[V\left(2 a_{s}, 0,0\right)-V\left(a_{s}, 0,0\right)\right]$. The renormalized anisotropy can then be defined as the ratio $\xi_{\text {meas }}=\Delta_{x t} / \Delta_{x y}$. Results for the renormalized anisotropy 4 are listed in Table 1 . 


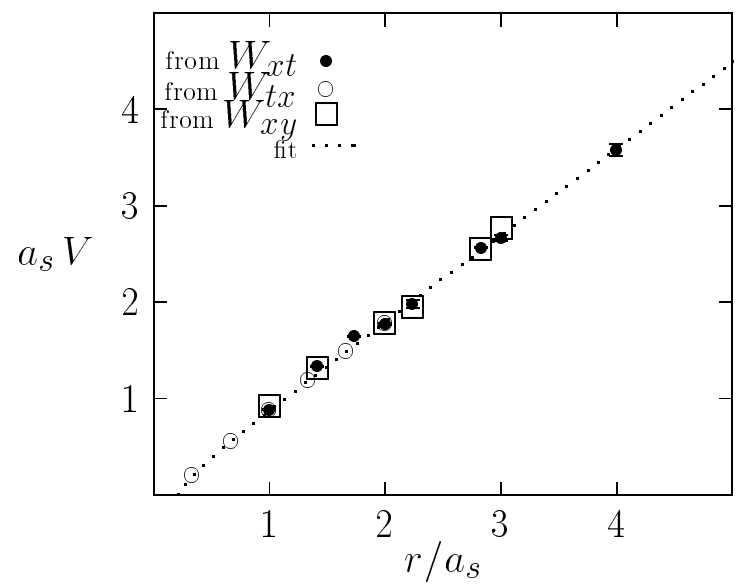

Figure 1. The static-quark potential from Wilson loops in different orientations. Results are shown for the action $S_{I I}$ with $\beta=2.0$ and $a_{t} / a_{s}=1 / 3$. The different potentials were shifted to agree at $r=a_{s}$.

Note that without tadpole improvement $\left(u_{s}=1\right)$, the renormalization of the anisotropy is a $20 \%$ effect; mean-field improvement reduces this renormalization to the $1-3 \%$ level. Results for the static-quark potential[ 1 are shown in Fig. 1 .

The masses of various low-lying glueballs were also calculated[3]. The results are shown in Fig. 2 as a function of the lattice spacing as measured in terms of the hadronic scale $r_{0}$. The scalar glueball mass from the improved action $S_{I I}$ exhibits dramatically reduced cutoff contamination compared to the Wilson action. Finite- $a_{s}$ errors are seen to be small for the tensor and pseudovector glueballs, although differences between the $E^{++}$ and $T_{2}^{++}$representations indicate violations of rotational invariance, especially for large $a_{s}$.

These results clearly demonstrate the usefulness of perturbatively-improved gluonic actions on anisotropic lattices. For coarse-lattice glueball studies, anisotropic lattices are crucial. The effectiveness of tadpole improvement in reducing the renormalization of the anisotropy to the level of a few per cent makes using anisotropic lattices no more difficult than isotropic ones. This work was supported by grants from the NSF, the DOE, and the NSERC of Canada.

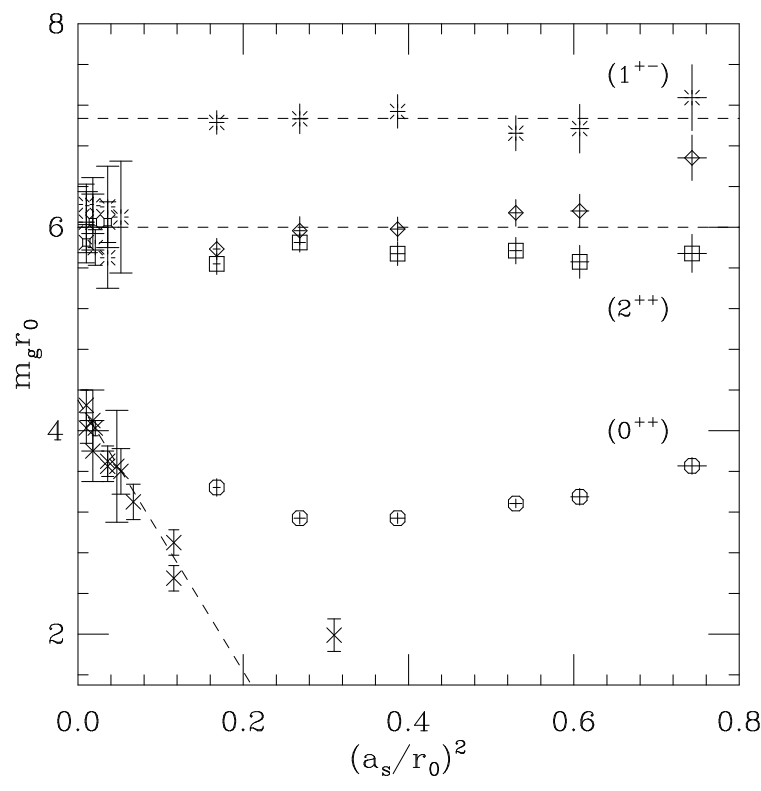

Figure 2. Low-lying glueball masses $m_{g}$ against lattice spacing $a_{s}$ in terms of the hadronic scale $r_{0} \approx 1 / 2 \mathrm{fm}$ defined by $\left[r^{2} d V(r) / d r\right]_{r=r_{0}}=1.65$ where $V(r)$ is the static quark potential. The $\times$ denote results for the scalar and tensor glueballs using the simple Wilson action [6]. Results using the improved action $S_{I I}$ for the $A_{1}^{++}, E^{++}, T_{2}^{++}$, and $T_{1}^{+-}$representations are indicated by $\circ, \square$, $\diamond$, and $*$, respectively. The dashed lines are linear fits to the Wilson results for the scalar and tensor; for the $1^{+-}$, the dashed line guides the eye.

\section{REFERENCES}

1. C. Morningstar and M. Peardon, Nucl. Phys. B (Proc. Suppl.) 47, 258 (1996).

2. M. Alford and T. Klassen, these proceedings.

3. C. Morningstar and M. Peardon, these proceedings.

4. M. Alford, T. Klassen, P. Lepage, C. Morningstar, M. Peardon, H. Trottier, to appear.

5. M. Lüscher and P. Weisz, Comm. Math. Phys. 97, 59 (1985).

6. J. Sexton, et al., Phys. Rev. Lett. 75, 4563 (1995); P. De Forcrand et al., Phys. Lett. B 152, 107 (1985); C. Michael and M. Teper, Nucl. Phys. B 314, 347 (1989); UKQCD Collaboration, Phys. Lett. B 309, 378 (1993). 